\title{
Uncovering the roles of oxygen vacancies in cation migration in lithium excess layered oxides $\dagger$
}

Cite this: Phys. Chem. Chem. Phys. 2014, 16, 14665

Received 25th April 2014

Accepted 4th June 2014

DOI: $10.1039 / c 4 c p 01799 d$

www.rsc.org/pccp

A novel oxygen vacancy assisted transition metal (TM) diffusion mechanism is proposed for the first time to explain the near-surface phase transformation in lithium excess transition metal layered oxides. Oxygen vacancies and TM migration have been observed at $\mathrm{nm}$ scale spatial resolution by Scanning Transmission Electron Microscopy and Electron Energy Loss Spectroscopy. Formation of (dilute) oxygen vacancies and their roles in assisting transition metal ion diffusion were further investigated using first principles calculations. The activation barriers of TM diffusion in the presence of oxygen vacancies are drastically reduced and consistently in a reasonable range for room temperature diffusion.

In spite of research on various kinds of materials in the past few decades, lithium intercalation compounds still provide the best performances as the cathode materials for lithium ion batteries (LIB). Among all candidates, the lithium-excess layered oxides have gained growing research interest in recent years. They can reach more than $250 \mathrm{~mA} \mathrm{~h} \mathrm{~g}^{-1}$ capacity with an average voltage of $4 \mathrm{~V}$, making them the candidates with highest energy densities among all known intercalation compounds for cathode materials. ${ }^{1-7}$ Being the source of major capacity improvement, the extra lithium ions, on the other hand, also cause unexplained phenomena in lithium-excess layered oxides. The rate performances of these materials are usually poor, and electron sources for the extra capacities have been under debate for years. ${ }^{4,8-11}$ In classical layered oxides, the transition metal (TM) oxide host $\mathrm{TMO}_{2}$ should retain its host structure during initial discharging/charging, while lithium ions are intercalated/de-intercalated from the host.

\footnotetext{
${ }^{a}$ Department of NanoEngineering, University of California San Diego, La Jolla, CA 92093, USA. E-mail: shirleymeng@ucsd.edu

${ }^{b}$ Department of Materials Science and Engineering,

Massachusetts Institute of Technology, Cambridge, MA, 02139, USA

${ }^{c}$ Center for Nanophase Materials Sciences, Oak Ridge National Laboratory, Oak Ridge, TN, 37831, USA

$\dagger$ Electronic supplementary information (ESI) available: STEM/EELS details, computational details, Ni diffusion barriers at Li15/28, neighboring TM valence change after oxygen vacancy formation at Li20/28, Mn diffusion barriers at Li20/28 and synthesis and sample preparation. See DOI: 10.1039/c4cp01799d
}

In contrast, recent studies suggested that in lithium-excess layered oxides, certain amounts of surface transition metal ions migrate from the transition metal layers to lithium layers during the very first charging-discharging cycle. ${ }^{12-17}$ These migrated ions are very stable in the lithium layers. They will likely block the lithium diffusion pathways and slow the lithium diffusion rates. Since the migrated surface transition metal ions could be one of the main factors that limit the material rate performances, and possibly cause instability in the voltage profile, it is crucial to understand their diffusion mechanism. Our previous work ${ }^{17-20}$ has suggested that the migration may be assisted by oxygen vacancies generated in the late charging states of the first cycle, but two critical questions remain unanswered: (1) how can we visualize the change in structure and chemical content at the atomic level and (2) what is the energy required for oxygen vacancies formation upon cycling and how do they assist transition metal migration. To answer these questions, a systematic study on generation of oxygen vacancies in Li-excess layered oxide $\mathrm{Li}\left[\mathrm{Li}_{1 / 6} \mathrm{Ni}_{1 / 6} \mathrm{Co}_{1 / 6} \mathrm{Mn}_{1 / 2}\right] \mathrm{O}_{2}$ was performed using Scanning Transmission Electron Microscopy/Electron Energy Loss Spectroscopy (STEM/EELS) experiments, and studies using the first principles computational method were performed on its analogue $\mathrm{Li}\left[\mathrm{Li}_{1 / 6} \mathrm{Ni}_{1 / 4} \mathrm{Mn}_{7 / 12}\right] \mathrm{O}_{2}$. EELS characterized the local oxygen and $\mathrm{TM}$ environments at the two phases and the interface region. The local diffusion barriers of TM ions were calculated in the presence of oxygen vacancies, and a hypothesis of the transition metal ion migration mechanism is proposed.

To visualize the material structural change upon cycling from the atomic level, aberration corrected high angle annular dark field (HAADF) STEM images of the material particles were obtained and are shown in Fig. 1 (left). Similar to previous observations, TM ions were found in the Li layer in the surface and subsurface regions, forming a defect spinel structure that is different from the layered structure in bulk. ${ }^{17}$ For quantitative analysis, EELS experiments were carried out on the phase transformation region (technical details in SI1, ESI $\dagger$ ). Spatially resolved EELS were obtained at a step of $\sim 0.6 \mathrm{~nm}$ from the surface to bulk and the spectra are presented in Fig. 1 (right). 


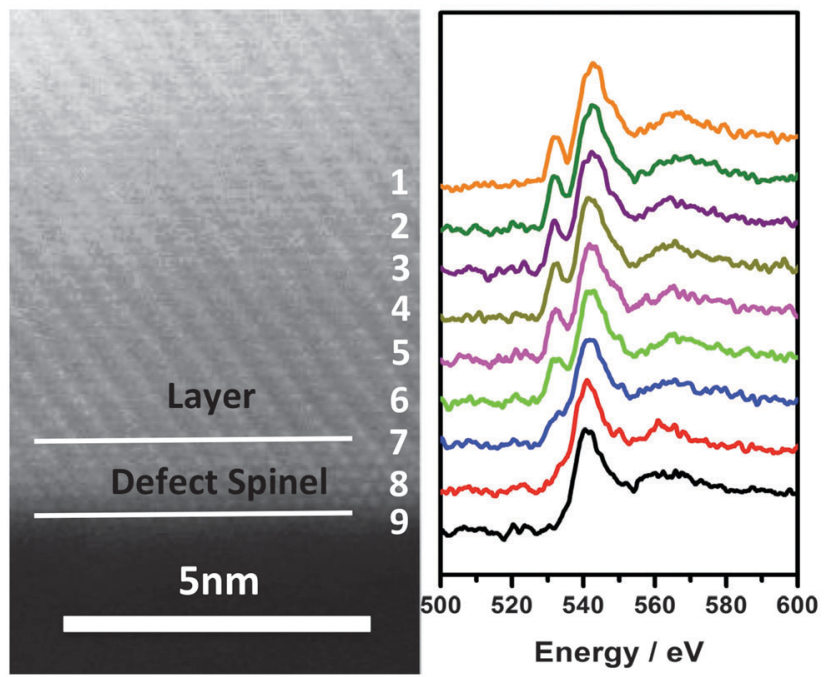

Fig. 1 Spatially resolved O K-edge EELS spectra from the bulk to the surface.

The data points are aligned with the STEM image to indicate where each spectrum was taken. For example, the black (data point 9) and red curves (data point 8) were acquired from the surface and near surface regions, the blue (data point 7) curve was acquired from the spinel-layered interface, and the orange (data point 1) curve was acquired from the bulk. The onset energy of the $\mathrm{O}$ K-edge pre-peak is aligned to $532 \mathrm{eV}$. Therefore, our analysis of the $\mathrm{O}$ K-edge is limited to the fine structures and not the chemical shift of the $\mathrm{O}$ K-edge. In Fig. 1, there is a clear $\mathrm{O}$ pre-peak in the spectra obtained in bulk. This pre-peak starts to decrease when it comes to the interface region of the spinel-like and layer phase, and disappears completely in the spinel-like phase near surface. The splitting of the $\mathrm{O}$ K-edge usually corresponds to the splitting of the TM 3d orbitals in a six-coordinated environment. The disappearance of the pre-peak can be ascribed to the following two main reasons: (1) reduction of neighboring TM and (2) oxygen vacancy formation, the splitting of neighbor TM 3d orbitals is no longer the same as six-coordinated. Usually the TM reduction comes along with oxygen vacancy formation due to charge compensation. In this case, there were synchrotron XRD studies showing oxygen loss in the late charging state. ${ }^{21}$ Therefore it is strongly evident that oxygen vacancies were present mostly at the surface region where the spinel-like phase formed, and gradually disappeared when it came to the bulk of the layer phase.

From the STEM/EELS results, it is clear that TM ion migration only happens in the region where the oxygen vacancies are present. First principles calculations were then carried out (detailed computational settings can be found in SI2, ESI $\dagger$ ) for deeper understanding of the material at the atomic level. As depicted in the inset of Fig. 2(a), each oxygen ion is bonded to 6 nearest cations. Three of them are Li ions in the lithium layer and the other three in the TM layer can be $\mathrm{Li}$, Ni or Mn. There are four different combinations of the three cations in the TM layer. These configurations are indicated by black circles in Fig. 2(a) and denoted as a, b, c and d. Oxygen vacancies can be located in these different local atomic configurations at different lithium
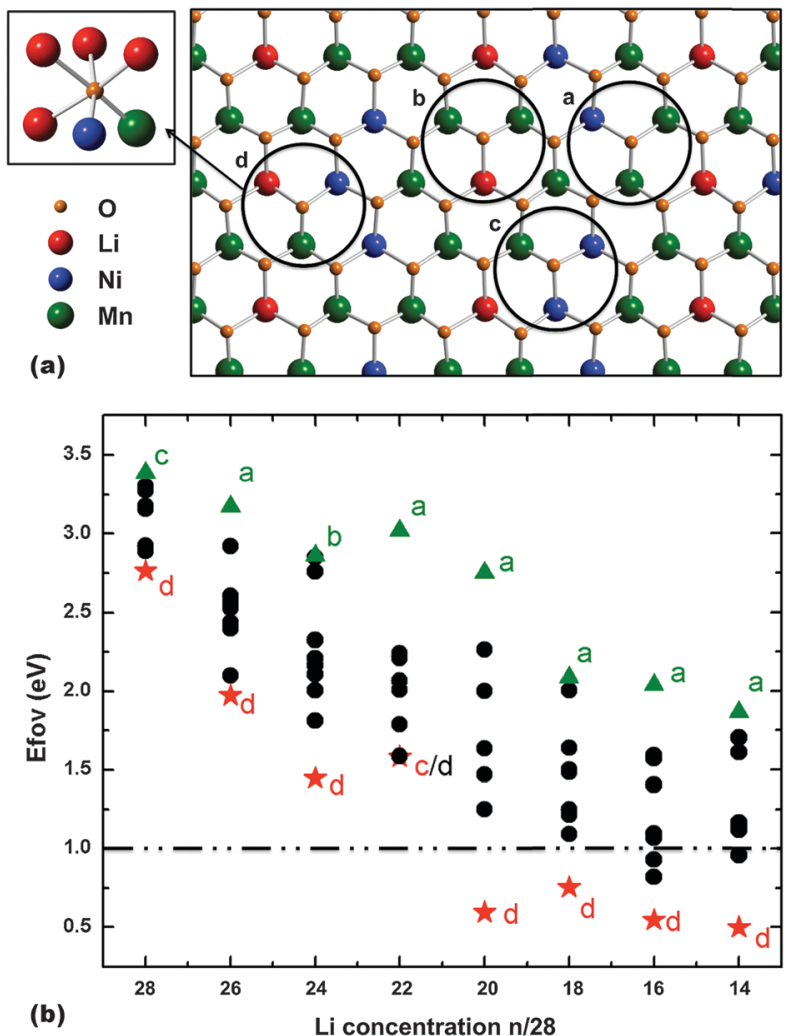

Fig. 2 (a) Atomic configurations of $\mathrm{Li}\left[\mathrm{Li}_{1 / 6} \mathrm{Ni}_{1 / 4} \mathrm{Mn}_{7 / 12}\right] \mathrm{O}_{2}$; (b) oxygen vacancy formation energy vs. Li concentration.

concentrations. More than 70 calculations were performed with oxygen vacancies in different atomic configurations. A general trend of calculated oxygen vacancy formation energies $\left(E_{\mathrm{fov}}\right)$ versus lithium concentrations is shown in Fig. 2(b).

The lithium concentration of these calculations covers from 28/28 (fully lithiated) to 14/28 (half delithiated), the range of which corresponds to the slopy region of the first charging voltage curve. It is clear that $E_{\mathrm{fov}}$ decreases sharply from $\sim 2.7 \mathrm{eV}$ in the fully lithiated state to less than $1 \mathrm{eV}$ for Li concentration between 20/28 to 14/28. Note that at Li concentration 20/28, the tetrahedral Li-Li dumbbells begin to form, leading to the possible formation of spinel-like phases. ${ }^{17}$ After that, $E_{\text {fov }}$ becomes relatively stable. Our data show a surprisingly consistent trend of $E_{\mathrm{fov}}$ in terms of local atomic configurations. $E_{\text {fov }}$ is usually low in configuration d while is usually high in configuration a. The results show a strong preference for certain local atomic environments for vacancy formation. Oxygen vacancies are more likely to form when their local environments are $\mathrm{Li}-\mathrm{Ni}-\mathrm{Mn}$ combinations in the transition metal layer. The electrostatic effect may partially contribute to this phenomenon. As in the metal oxides, oxygen vacancies exhibit positive charges (Kroger notation $\mathrm{V}_{\mathrm{o}}^{*}$ ) therefore should be more stable near the cations with less positive charges. In this work, all calculations were bulk calculations, while in reality, it is easier to form oxygen vacancies at material surfaces or sub-surfaces than in the bulk.

In order to investigate the migration mechanism of TM ions, the transition state theory is adopted in this work, as it has been extensively utilized to describe the cation diffusion mechanism 
in the materials. ${ }^{22}$ In $\mathrm{Li}_{x} \mathrm{Ni}_{1 / 4} \mathrm{Mn}_{7 / 12} \mathrm{O}_{2}$, both $\mathrm{Ni}$ and $\mathrm{Mn}$ ions are located originally in the octahedral site in the TM layer. An empty tetrahedral site in the Li layer is face-sharing with the TM octahedron. The shared-face is composed of three oxygen ions forming a triangular oxygen plane. When the three $\mathrm{Li}$ ions near the empty tetrahedral site are extracted, the TM ion may be able to migrate from the octahedral site to the empty tetrahedral site through the shared oxygen plane and then migrate to another octahedral site. If no oxygen vacancies are present, the diffusion barriers for TM ions are usually high $\left(0.7-0.8 \mathrm{eV}\right.$ for $\mathrm{Ni}^{2+}, 2.6 \mathrm{eV}$ for $\mathrm{Mn}^{4+}$, comparable to previous studies ${ }^{23}$ ), but the barriers can be significantly reduced when oxygen vacancies are introduced. The possible reasons of the large differences between $\mathrm{Ni}^{2+}$ and $\mathrm{Mn}^{4+}$ diffusion barriers are suspected to be their electronic structure differences as well as the higher charge of $\mathrm{Mn}^{4+}$.

$\mathrm{Ni}$ diffusion from initial octahedral sites in the TM layer to the nearest tetrahedral sites in the $\mathrm{Li}$ layer was investigated at three different $\mathrm{Li}$ concentrations: $\mathrm{Li}_{25 / 28} \mathrm{Ni}_{1 / 4} \mathrm{Mn}_{7 / 12} \mathrm{O}_{2}$, $\mathrm{Li}_{20 / 28} \mathrm{Ni}_{1 / 4} \mathrm{Mn}_{7 / 12} \mathrm{O}_{2}$ and $\mathrm{Li}_{15 / 28} \mathrm{Ni}_{1 / 4} \mathrm{Mn}_{7 / 12} \mathrm{O}_{2}$ (shown in SI3, $\mathrm{ESI} \dagger)$. Three different Ni migration pathways are investigated when oxygen vacancies are introduced (shown in Fig. 3): oxygen vacancies present (1) in the shared oxygen plane between the octahedral site and the tetrahedral site; (2) in the octahedral vertex but not in the shared oxygen plane; and (3) in the tetrahedral vertex but not in the shared oxygen plane. Although the exact values of $\mathrm{Ni}$ diffusion barriers are different for different diffusion paths, the trend is consistent over all $\mathrm{Li}$ concentrations. Therefore only the results for $\mathrm{Li}_{20 / 28} \mathrm{Ni}_{1 / 4} \mathrm{Mn}_{7 / 12} \mathrm{O}_{2}$ are discussed below as the representative case. After the introduction of oxygen vacancies, the neighboring $\mathrm{Ni}$ will be reduced (SI4, ESI $\dagger$ ), therefore, the diffusion barrier of $\mathrm{Ni}^{2+}$ ions was investigated for a consistent comparison. Fig. 3 shows the calculated Ni diffusion barriers at $\mathrm{Li}_{20 / 28} \mathrm{Ni}_{1 / 4} \mathrm{Mn}_{7 / 12} \mathrm{O}_{2}$ with oxygen vacancies in different locations mentioned above. It is clear that the locations of oxygen vacancies have a significant impact on the Ni diffusion barriers. When $\mathrm{Ni}$ diffuses through the shared oxygen plane with vacancies, although the oxygen electron charge density may be less dense, the $\mathrm{Ni}$ diffusion barriers are around $1 \mathrm{eV}$, which are slightly higher than

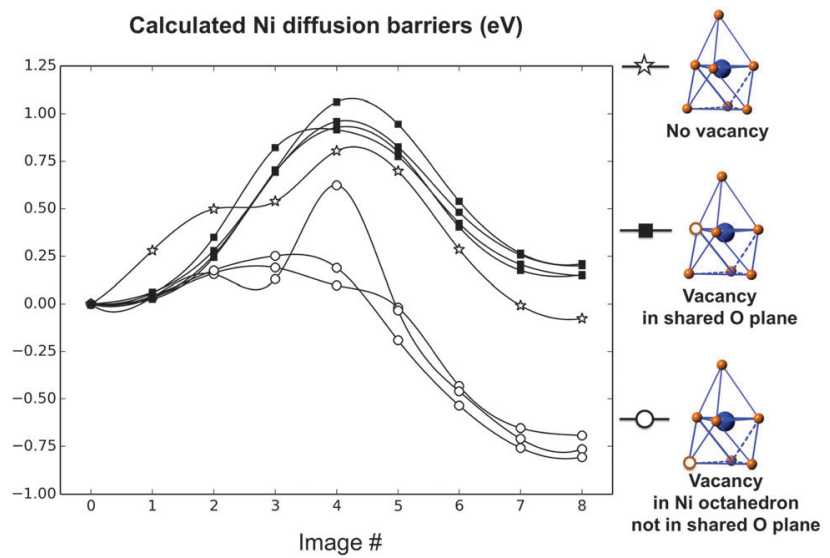

Fig. 3 Calculated Ni diffusion barriers with oxygen vacancies in different positions at $\mathrm{Li}_{20 / 28} \mathrm{Ni}_{1 / 4} \mathrm{Mn}_{7 / 12} \mathrm{O}_{2}$ (vacancies in the tetrahedron but not in the shared plane are unstable). the barriers without oxygen vacancies. When Ni migrates from the five-coordinated octahedral site, it will be much more unstable in the regular tetrahedral site and Ni diffusion barriers are reduced to as low as $0.2 \mathrm{eV}$ to $0.5 \mathrm{eV}$. When the vacancies appear at the tetrahedral vertex, which is not in the shared oxygen plane, the three-coordinated tetrahedral site becomes stable with a significant valence change of nearby transition metal ions. In this case, calculated $\mathrm{Ni}$ diffusion barrier is a mixing of barriers arising from $\mathrm{Ni}$ ion migration and charge transfer between ions, therefore it is not included in Fig. 3. A study on $\mathrm{Mn}$ diffusion was also performed at $\mathrm{Li}_{20 / 28} \mathrm{Ni}_{1 / 4} \mathrm{Mn}_{7 / 12} \mathrm{O}_{2}$, and similar observations were made (shown in SI5, ESI $\dagger$ ). With no oxygen vacancies, $\mathrm{Mn}$ is much more stable in the octahedral site than in the tetrahedral site, and the barrier is as high as $2.6 \mathrm{eV}$. However, when oxygen vacancies are introduced in the octahedral site but not in the shared plane, the diffusion barrier can be reduced to $\sim 0.7 \mathrm{eV}$. Besides, if oxygen vacancies are introduced in the tetrahedral site but not the shared plane, Mn becomes very unstable in the three-coordinated tetrahedral site and will automatically diffuse to the nearby octahedral site with no barrier. How oxygen vacancies would assist TM migration is shown quantitatively for the first time from computations; our findings reveal that in the presence of oxygen vacancies both $\mathrm{Ni}$ and $\mathrm{Mn}$ have the potential to diffuse at room temperature and Ni has lower barrier, while the experimental evidence needs future investigation.

\section{Conclusions}

Combining both STEM/EELS experimental observations and first principles computational studies, we demonstrated that in the Li-excess materials oxygen vacancies are present in these regions and assist the TM ion migration through a novel mechanism proposed below: during first cycle charging, after most TM ions are fully oxidized, oxygen ions start to participate in the oxidation process (lose electron) and oxygen vacancies would form with a formation energy about 0.5-0.6 eV. Possibly due to the slow oxygen migration, oxygen vacancies mostly form near the material surfaces and sub-surfaces with 5-6 atomic layers. A significant fraction of the TM ions in these regions therefore are subjected to five (or even less) O-coordination due to the presence of either oxygen vacancies or the broken TM-O bonds on the particle surfaces. TM ions in those five (or less) O-coordinated defect polyhedral sites become much unstable and will spontaneously migrate to the fully-coordinated polyhedral sites nearby in the Li layer. On the other hand, although many of the $\mathrm{Li}$ ions are extracted upon charging, stable $\mathrm{Li}-\mathrm{Li}$ dumbbells are also formed leaving only certain sites in Li layers that are available for TM occupation. Combination of the above two reactions therefore causes the formation of a spinel-like phase from the material surface towards the bulk until reaching a region (2-3 $\mathrm{nm}$ beneath the surface after the first cycle) where oxygen vacancy concentrations are too low to assist the TM ion migration. It was reported that although it might be subjected to a further phase change, this TM-ion-migrated region is present for 50 cycles with its thickness unchanged. ${ }^{24}$ Our work rationalizes 
these observations and sheds light on the atomic level structural change upon cycling in this class of high energy density cathode materials. The critical role of oxygen ions must be considered for further optimization of this class of materials. Surface coating or doping elements with stronger affinity to oxygen is expected to reduce the phase transformation.

\section{Acknowledgements}

The authors acknowledge the financial support from the Assistant Secretary for Energy Efficiency and Renewable Energy, Office of Vehicle Technologies of the U.S. DOE under Contract No. DE-AC0205CH11231, Subcontract No. 7056412 under the Batteries for Advanced Transportation Technologies (BATT) Program. Computing resources are provided by Extreme Science and Engineering Discovery Environment (XSEDE) supported by National Science Foundation grant number OCI-1053575. The STEM/EELS work was carried out through a user project supported by ORNL's Center for Nanophase Materials Sciences (CNMS), which is sponsored by the Scientific User Facilities Division, Office of Basic Energy Sciences, US Department of Energy. We thank Mr Haodong Liu for sample preparation and Dr S. Curtarolo and coworkers for providing aconvasp code.

\section{References}

1 W. S. Yoon, S. Iannopollo, C. P. Grey, D. Carlier, J. Gorman, J. Reed and G. Ceder, Electrochem. Solid-State Lett., 2004, 7, A167-A171.

2 Y. S. Meng, G. Ceder, C. P. Grey, W. S. Yoon, M. Jiang, J. Breger and Y. Shao-Horn, Chem. Mater., 2005, 17, 2386-2394.

3 Z. H. Lu, D. D. MacNeil and J. R. Dahn, Electrochem. Solid-State Lett., 2001, 4, A191-A194.

4 Z. H. Lu and J. R. Dahn, J. Electrochem. Soc., 2002, 149, A815-A822.

5 C. H. Lei, J. Bareno, J. G. Wen, I. Petrov, S. H. Kang and D. P. Abraham, J. Power Sources, 2008, 178, 422-433.

6 C. R. Fell, K. J. Carroll, M. F. Chi and Y. S. Meng, J. Electrochem. Soc., 2010, 157, A1202-A1211.
7 J. Breger, M. Jiang, N. Dupre, Y. S. Meng, Y. Shao-Horn, G. Ceder and C. P. Grey, J. Solid State Chem., 2005, 178, 2575-2585.

8 M. M. Thackeray, S. H. Kang, C. S. Johnson, J. T. Vaughey, R. Benedek and S. A. Hackney, J. Mater. Chem., 2007, 17, 3112-3125.

9 A. D. Robertson and P. G. Bruce, Electrochem. Solid-State Lett., 2004, 7, A294-A298.

10 M. Jiang, B. Key, Y. S. Meng and C. P. Grey, Chem. Mater., 2009, 21, 2733-2745.

11 A. R. Armstrong, M. Holzapfel, P. Novak, C. S. Johnson, S. H. Kang, M. M. Thackeray and P. G. Bruce, J. Am. Chem. Soc., 2006, 128, 8694-8698.

12 J. Liu and A. Manthiram, J. Mater. Chem., 2010, 20, 3961-3967.

13 S. T. Myung, K. Izumi, S. Komaba, Y. K. Sun, H. Yashiro and N. Kumagai, Chem. Mater., 2005, 17, 3695-3704.

14 B. C. Park, H. B. Kim, S. T. Myung, K. Amine, I. Belharouak, S. M. Lee and Y. K. Sun, J. Power Sources, 2008, 178, 826-831.

15 J. Hong, H. D. Lim, M. Lee, S. W. Kim, H. Kim, S. T. Oh, G. C. Chung and K. Kang, Chem. Mater., 2012, 24, 2692-2697.

16 M. Gu, I. Belharouak, A. Genc, Z. G. Wang, D. P. Wang, K. Amine, F. Gao, G. W. Zhou, S. Thevuthasan, D. R. Baer, J. G. Zhang, N. D. Browning, J. Liu and C. M. Wang, Nano Lett., 2012, 12, 5186-5191.

17 B. Xu, C. R. Fell, M. F. Chi and Y. S. Meng, Energy Environ. Sci., 2011, 4, 2223-2233.

18 C. R. Fell, M. F. Chi, Y. S. Meng and J. L. Jones, Solid State Ionics, 2012, 207, 44-49.

19 K. J. Carroll, D. Qian, C. R. Fell, S. Calvin, G. M. Veith, M. Chi, L. Baggetto and Y. S. Meng, Phys. Chem. Chem. Phys., 2013, 15, 11128.

20 C. R. Fell, D. Qian, K. J. Carroll, M. Chi, J. L. Jones and Y. S. Meng, Chem. Mater., 2013, 25, 1621-1629.

21 C. R. Fell, D. Qian, K. J. Carroll, M. Chi, J. L. Jones and Y. S. Meng, Chem. Mater., 2013, 25, 1621.

22 A. Van der Ven and G. Ceder, Electrochem. Solid-State Lett., 2000, 3, 301-304.

23 J. Reed and G. Ceder, Chem. Rev., 2004, 104, 4513-4534.

24 A. Boulineau, L. Simonin, J. F. Colin, C. Bourbon and S. Patoux, Nano Lett., 2013, 13, 3857-3863. 